\title{
Bell's palsy: Our experience and review of 30 cases
}

\author{
Ratna Priya, Seema Monga*, Junaid Malik, Arun P Sharma, Humra Shamim, Shahid Rasool and Khaja Naseeruddin \\ Department of ENT, HAH Centenary Hospital and Hamdard Institute of Medical Sciences and Research, New Delhi, India
}

\begin{abstract}
Bell's palsy [BP], named after Sir Charles Bells, is defined as acute onset peripheral facial nerve paralysis that is idiopathic, comprising of about 70\% of the usual facial palsy cases. Many controversies exist about the exact diagnostic protocol and treatment options for Bell's palsy, but most commonly followed treatment options are corticosteroids with or without acyclovir, acupuncture, physiotherapy.

In our study we are presenting clinicopathological 30 cases of BP with details regarding age of presentation, site of palsy, onset, and grade of paresis, treatment and sequelae. The purpose is to look over clinical characteristics of Bell's palsy to help provide information regarding the disease in our hospital setting and correlation with other similar studies in literature.
\end{abstract}

\section{Introduction}

Bell's palsy [BP], named after Sir Charles Bells, is defined as acute onset peripheral facial nerve paralysis that is idiopathic, comprising of about $70 \%$ of the usual facial palsy cases. Epidemiological surveys have reported the incidence varying from 10-40 person 10,000 individuals in different regions [1]. Mostly it is unilateral but even bilateral cases have been rarely reported [2]. In spite of the fact that Bell's palsy is seen across a large age group, the incidence was commonly observed to be the highest in the 15- to 45 -year-old patients [3]. Multifactorial aetiology has been found to be associated with this uncommon condition including physiological compression of the nerve because of arteriospasm, venous congestion or ischemia, and anatomical narrowing of the bony canal. Quite a few cases have come up that do support a familial tendency implying the inheritance of aberrant facial canal [4]. Pregnancy is a well-known risk factor, usually during the third trimester and early postpartum periods, with incidence as high as 3 times greater when compared to the general population [5]. Other risk factors for BP include hypertension, diabetes, obesity, severe preeclampsia and upper respiratory tract ailments [6].

In around $80 \%$ of cases, it resolves with complete recovery [7], however in a few cases it can have debilitating consequences like exposure keratitis, vision loss, permanent facial paresis, social distress, and social alienation. Many controversies exist about the exact diagnostic protocol and treatment options for Bell's palsy, but most commonly followed treatment options are corticosteroids with or without acyclovir, acupuncture, physiotherapy.

In our study we are presenting clinicopathological 30 cases of $\mathrm{BP}$ with details regarding age of presentation, site of palsy, onset, and grade of paresis, treatment and sequelae.

\section{Materials and methods}

This is an observational study of 30 patients of Bell's palsy who presented to the department of ENT at Hamdard Institute of Medical Sciences and Research, Jamia Hamdard, New Delhi from January 2018 to January 2019. The study included all patients who had developed unilateral idiopathic lower motor neuron palsy. The grade of palsy was assessed using House Brackmann grading system. Candidates with history of otitis media, neurological illness or any trauma to the head and neck were excluded from the study.

A thorough medical history was compiled including age, duration of palsy and type of onset whether being sudden or gradual. Other complaints such as preceding earache, any upper respiratory tract infection or rashes were also looked for. Along with this, history related to comorbidities such as diabetes mellitus, hypertension or any other illness was also asked.

A complete examination of pinna, external auditory canal and tympanic membrane was done. A proper cranial nerve examination was performed to rule out other nerve's involvement if any. Systemic examination was also done.

All the patients were prescribed oral prednisolone dose being 1 $\mathrm{mg} / \mathrm{kg}$ for 5 days followed by gradual tapering over a period of two weeks along with five-day course of acyclovir $800 \mathrm{mg}$ five times a day for three days. Facial massage and exercises were explained to the patients. Trans electric nerve stimulation (TENS) was also advised along with medicines to hasten recovery in all patients. These patients were followed up weekly up to 6 weeks and recovery was analyzed using House-Brackmann scale.

\section{Results}

In our series, we had 16 male and 14 female patients (Table 1). Mean age of patients was 33 years varying from 13 -year-old to 55 -year-old. In age distribution maximum patients were in the 40-50-year age group (Table 2). Among our patients, 15 had left sided palsy and the remaining had right sided palsy. In our group, as per the House-Brackmann (HB)

${ }^{*}$ Correspondence to: Seema Monga, Department of ENT, HAH Centenary Hospital and Hamdard Institute of Medical Sciences and Research, New Delhi, India, Tel: 8470040230; E-mail: dr.seema.monga@gmail.com

Key words: Bell's palsy, House-Brackmann grading, corticosteroids, antiviral Received: April 05, 2019; Accepted: April 17, 2019; Published: April 19, 2019 
grading system (Table 3), 13 patients had grade 2 LMN palsy,6 patients had grade 3 LMN palsy, 10 patients have grade 4 palsy and only one presented with grade $5 \mathrm{LMN}$ palsy. Hence, the highest incidence as far as severity was concerned was of HB grade II (Table 4).

On further enquiry, earache was the most prominent associated symptom being seen 13 out of 30 patients. Upper respiratory tract infection preceding bell's palsy was found in 3 cases. One of our patients had vesicles over the pinna along with bell's palsy. Two patients presented with BP in second trimester.

All the patients were treated with $1 \mathrm{mg} / \mathrm{kg}$ prednisolone for 5 days with tapering over two weeks, except the two pregnant patients.26/30 patients had complete recovery following treatment (Table 5) [8]. Among our patients, 18 of them had onset of recovery in the second week, 7 patients had recovery in the third follow up week. Only one patient had onset of recovery in the $4^{\text {th }}$ week. Six patients had incomplete recovery. After complete treatment with steroids and acyclovir, one of the patients had complete recovery in $3^{\text {rd }}$ week, 12 patients had complete recovery in $4^{\text {th }}$ week, 3 patients recovered completely in the $5^{\text {th }}$ week and 10 patients had complete recovery in 6 weeks. Cases with incomplete recovery further underwent HRCT Temporal bone with CT brain to rule out facial neuroma.

Table 1. Age distribution

\begin{tabular}{|c|c|c|}
\hline Age (years) & No. of cases & Percentage (N= 30) \\
\hline $10-20$ & 2 & $7 \%$ \\
\hline $20-30$ & 10 & $33 \%$ \\
\hline $30-40$ & 7 & $23 \%$ \\
\hline $40-50$ & 6 & $20 \%$ \\
\hline $50-60$ & 5 & $17 \%$ \\
\hline
\end{tabular}

Table 2. Sex distribution

\begin{tabular}{|c|c|c|}
\hline Sex & Number of cases & Percentage (Total number of cases =30) \\
\hline Male & 16 & $53 \%$ \\
\hline Female & 14 & $47 \%$ \\
\hline
\end{tabular}

Table 3. House-Brackmann scale ranges between I (normal) and VI (no movement) [18].

\begin{tabular}{|c|c|}
\hline HB grading & Description \\
\hline I & Normal symmetrical function \\
\hline II & $\begin{array}{l}\text { Slight weakness noticeable only on close inspection } \\
\text { Complete eye closure with minimal effort } \\
\text { Slight asymmetry of smile with maximal effort } \\
\text { Synkinesis barely noticeable, contracture, or spasm absent }\end{array}$ \\
\hline III & $\begin{array}{l}\text { Obvious weakness, but not disfigurment } \\
\text { May not be able to lift eyebrows } \\
\text { Complete eye closure and strong but asymmetrical mouth movement } \\
\text { Obvious, but not disfiguring synkinesis, mass movement, or spasm }\end{array}$ \\
\hline IV & $\begin{array}{l}\text { Obvious disfiguring weakness } \\
\text { Inability to lift eyebrows } \\
\text { Incomplete eye closure and asymmetry of mouth with maximal effort } \\
\text { Severe synkinesis, mass movement, or spasm }\end{array}$ \\
\hline $\mathrm{V}$ & $\begin{array}{l}\text { Motion barely perceptible } \\
\text { Incomplete eye closure, slight movement at mouth corner } \\
\text { Synkinesis, contracture, and spasm usually absent }\end{array}$ \\
\hline VI & No movement, loss of tone, no synkinesis, contracture, or spasm \\
\hline
\end{tabular}

Table 4. Distribution of patients according to House Brackmann grading.

\begin{tabular}{|c|c|c|}
\hline HB Grade & No. of patients $(\mathbf{n}=\mathbf{3 0})$ & Percentage \\
\hline I & & \\
\hline II & 13 & $43 \%$ \\
\hline III & 6 & $20 \%$ \\
\hline IV & 10 & $33 \%$ \\
\hline V & 1 & $4 \%$ \\
\hline VI & & \\
\hline
\end{tabular}

Table 5. Recovery following treatment.

\begin{tabular}{|c|c|c|}
\hline Treatment Outcome & No. of patients & Percentage (n=30) \\
\hline Complete Recovery & 26 & $87 \%$ \\
\hline Incomplete recovery & 4 & $13 \%$ \\
\hline
\end{tabular}

\section{Discussion and conclusion}

The history of Bell's palsy can be traced back to $18^{\text {th }}$ century, when Professor Nicolaus Friedrich, described and published his clinical findings, De paralysis MusculorumFaciei Rheumatica [9].

Bell's palsy is the most common (around 70\%) cause of facial palsy [1]. Although exact etiopathogenesis is still not completely known but viral aetiology leading to facial nerve compression due to oedema is strongly suspected. The usual site of pathology seems to be the labyrinthine segment of facial nerve starting at the meatal foramen. The meatal foramen is at the lateral internal auditory canal where the labyrinthine segment of the facial nerve exits the internal auditory canal and rises to the geniculate ganglion [10,11]. Other cause of facial palsy like stroke, trauma, brain tumour, parotid gland tumour or trauma, malignancies involving the facial nerve, herpes zoster infection, otitis media causing severe nerve inflammation, sarcoidosis should be ruled out to diagnose Bell's palsy.

Most common age of presentation is 15 to $45 \mathrm{yr}$ and in our study mean age of presentation was 33 years only 1 patient out of 30 cases was a child.

Male to female ratio in our study 1.14 which is accordance with most of the study while in a study done by Cirpaciu et al. [12], there was a female predominance. Monini et al. [13], reported (B) that peak age of incidence was $50 \mathrm{yrs}$ around $53.7 \%$ in this study were male in the study done.

Patients with BP usually progress from beginning of symptoms to maximal weakness within 3 days and almost always within 7 days. A more gradual onset or progression over than 14 days is unlikely to be due to Bell's Palsy. Even in our study mean duration of appearance of maximum weakness was 4 days [13].

Majority of our patients was having grade 2 House Brackmann facial paresis which is similar to the studies done by Cirpaciu et al. [12]. There is no prediction for either side of the face as reported by lot of studies although reported that Right side was more frequently involved than left side in his study. In our study, similarly both sides were equally affected.

Through history and detailed examination to rule out other causes of facial paresis is the most important aspect of making the diagnosis of BP. History should include history of recent rashes, arthralgia, and fever, exposure to influenza vaccine, ticks, or visit to areas where Lyme disease is endemic.

Unnecessary lab testing \& imaging is usually not recommended in these patients. It is generally suggested that unless complete facial palsy is present, electro diagnostic testing provides no clinical benefit. Even in our study we did not prescribe routine lab testing in any of these patients and imaging (MRI) was done only in those 4 patients who did not improve even after 6 weeks of treatment

Bell's Palsy has been a diagnosis of exclusion, but several studies have been demonstrated a link to HSV [14], but most of the studies have made a diagnosis of Bell's Palsy based on history and examination. Even in our study serological testing of HSV could not be performed due to financial restraints. 
There are many co morbid conditions associated with BP like diabetes mellitus, pregnancy, immunocompromised condition, influenza. In our study 1 patient had diabetes, 2 were pregnant and 1 was hypertensive. Valenca et al. [15] and Glass et al. [16] reported a high risk for pregnant women to develop Bell's palsy [15] while Cirpaciu et al. [12] reported that none of their BP patients were pregnant or in puerperium.

An important concern for all Bell's palsy patients is to know chances of improvement of bell's palsy therefore careful counselling \& reassurance of patients should be done. Chances of complete recovery with Bell's palsy are very high around $70 \%$ with no treatment \& $90 \%$ with steroids. In our study $87 \%$ recovered completely and four patients (13\%) who did not recover were further investigated but no other medical cause of facial palsy could not find in these patients

The most popular treatment protocol includes corticosteroids along with antivirals. Prednisolone is mostly given in a 10-day tapering course starting with $60 \mathrm{mg}$ per day in adults. Because of possibility of HSV-1 as an etiological factor and also due to minimal side effect of either Acyclovir $400 \mathrm{mg} 5$ times a day for 7 days or valcyclovir $1 \mathrm{gm}$ can be given 3 times a day for 7 days. These medicines are most effective if given within 3 days of onset of symptoms. In addition to steroids and antivirals, all patients in our study were offered effective counselling, physiotherapy and eye care as the disease may lead to both early and late manifestations such as ineffective eye closure, dryness predisposing to corneal ulcer and loss of sight. However, all these early sequelae can be avoided with proper eye care. Unfortunately, incomplete recovery of facial nerve leading to permanent disfigurement has a negative impact on the quality of life of patient and may even cause clinical depression and social withdrawal [15]. Hence, I would like to emphasize that supportive treatment including physiotherapy and facial massage and exercise along with mirror image rehabilitation play their part to keep the paralyzed facial muscles in satisfactory state.

The likelihood cause of facial paralysis in bells is due to compression of nerve at the labyrinthine segment starting at the meatal foramen [17]. The meatal foramen is at the lateral internal auditory canal where the labyrinthine segment of the facial nerve leaves the internal auditory canal and rises to the geniculate ganglion. Nowadays, there is little evidence supporting Surgical decompression of the facial nerve as a result of certain limitations including few case reports, methodological inadequacies, reporting bias, and safety problems [18].

A lot of authors have reported that Bell's palsy in children can be effectively managed with physiotherapy and multivitamin alone thus avoiding side effects of steroids. In our study as well, child was managed just with physiotherapy and multivitamins and showed complete recovery in 3 weeks.

Patients who take longer than 10 weeks to demonstrate first signs of recovery often have a lot of synkinesis later as do those with complete flaccid paralysis within 24 hours of symptom onset. During the follow up period of 6 months we did not observe any such synkinesis in our four patients who did not recover completely even in six months.
Unfortunately, we could not follow up these patients for a longer period to observe any long-term sequelae.

Patients who had one episode of Bell's palsy have an $8 \%$ chance of recurrence $(G)$. In our study only one patient had recurrent episodes of Bell's palsy, around three episodes in six months.

Hence, to summarize Bell's palsy though not a life-threatening condition but has significant functional and psychological impact. Majority of cases do undergo spontaneous remission, still the mainstay for treatment remains oral steroids and acyclovir except in those patients where these drugs are contraindicated. Supportive care in the form of eye care, physiotherapy and facial exercises also play a significant part in early recovery.

\section{References}

1. De Diego-Sastre JI, Prim-Espada MP, Fernández-García F (2005) The epidemiology of Bell's palsy. Rev Neurol 41: 287-290. [Crossref]

2. Arias G, Nogués J, Mañós M, Amilibia E, Dicenta M (1998) Bilateral facial nerve palsy: four case reports. ORL J Otorhinolaryngol Relat Spec 60: 227-229. [Crossref]

3. Peitersen E (2002) Bell's palsy: the spontaneous course of 2,500 peripheral facial nerve palsies of different etiologies. Acta Otolaryngol Suppl: 4-30. [Crossref]

4. Olsen KD (1984) Facial nerve paralysis 1. General evaluation, Bell's palsy. Postgrad Med 75: 219-225. [Crossref]

5. Shafer WG, Hine MK, Levy BM (1983) A Textbook of Oral Pathology. (4 $4^{\text {th }}$ edn), WB Saunders Co, Philadelphia, pp: 859-860.

6. Hilsinger RL Jr, Adour KK, Doty HE (1975) Idiopathic facial paralysis, pregnancy, and the menstrual cycle. Ann Otol Rhinol Laryngol 84: 433-442. [Crossref]

7. Gantz BJ, Gmür A, Fisch U (1982) Intraoperative evoked electromyography in Bell's palsy. Am J Otolaryngol 3: 273-278. [Crossref]

8. House JW, Brackmann DE (1985) Facial nerve grading system. Otolaryngol Head Neck Surg 93: 146-147. [Crossref]

9. Savadi-Oskouei D, Abedi A, Sadeghi-Bazargani H (2008) Independent role of hypertension in Bell's palsy: a case-control study. Eur Neurol 60: 253-257. [Crossref]

10. Bird TD (1979) Nicolaus A. Friedreich's description of peripheral facial nerve paralysis in 1798. J Neurol Neurosurg Psychiatry 42: 56-58. [Crossref]

11. Fisch U, Esslen E (1972) Total intratemporal exposure of the facial nerve: pathologic findings in Bell's palsy. Arch Otolaryngol 95: 335-341. [Crossref]

12. Tiemstra JD, Khatkhate N (2007) Bell's palsy: diagnosis and management. Am Fam Physician 76: 997-1002. [Crossref]

13. Unuvar E, Oguz F, Sidal M, Kilic A (1999) Corticosteroid treatment of childhood Bell's palsy. Pediatr Neurol 21: 814-816. [Crossref]

14. Cirpaciu D, Goanta CM, Cirpaciu MD (2014) Recurrences of Bell's palsy. J Med Life 7 Spec No 3: 68-77. [Crossref]

15. Monini S, Lazzarino AI, Iacolucci C, Buffoni A, Barbara M (2010) Epidemiology of Bell's palsy in an Italian Health District: incidence and case-control study. Acta Otorhinolaryngol Ital 30: 198. [Crossref]

16. Valença MM, Valença LP, Lima MC (2001) Idiopathic facial paralysis (Bell's palsy): a study of 180 patients. Arq Neuropsiquiatr 59: 733-739. [Crossref]

17. Valente SM (2004) Visual disfigurement and depression. Plast Surg Nurs 24: 140-146. [Crossref]

18. Gantz BJ, Rubinstein JT, Gidley P, Woodworth GG (1999) Surgical management of Bell's palsy. Laryngoscope 109: 1177-1188. [Crossref]

Copyright: (C2019 Priya R. This is an open-access article distributed under the terms of the Creative Commons Attribution License, which permits unrestricted use, distribution, and reproduction in any medium, provided the original author and source are credited. 